\title{
Charge Pumping in Carbon Nanotubes
}

\author{
P. J. Leek, M. R. Buitelaar, V. I. Talyanskii, C. G. Smith, D. Anderson, and G. A. C. Jones \\ Cavendish Laboratory, University of Cambridge, Madingley Road, Cambridge, CB3 OHE, United Kingdom
}

\author{
J. Wei and D. H. Cobden
}

Department of Physics, University of Washington, Seattle, Washington 98195, USA

(Received 20 July 2005; published 13 December 2005)

\begin{abstract}
We demonstrate charge pumping in semiconducting carbon nanotubes by a traveling potential wave. From the observation of pumping in the nanotube insulating state we deduce that transport occurs by packets of charge being carried along by the wave. By tuning the potential of a side gate, transport of either electron or hole packets can be realized. Prospects for the realization of nanotube based singleelectron pumps are discussed.
\end{abstract}

DOI: 10.1103/PhysRevLett.95.256802

The phenomenon of charge pumping has attracted considerable interest in the last two decades from both fundamental and applied points of view [1-10]. In pumping, a periodic in time and spatially inhomogeneous external perturbation yields a dc current. If a fixed number $n$ of electrons is transferred during a cycle then the pumping current is quantized in units of $e f$, where $e$ is the electron charge and $f$ is the perturbation frequency. An important aspect of single-electron pumps is their potential to provide an accurate frequency-current conversion which could close the measurement triangle relating frequency, voltage, and current. Previously, a realization of quantized current $I=n e f$ has been achieved in two different ways: first, using devices comprising charge islands and controlled by a number of phase-shifted ac signals $[3,4,7]$; and second, using one-dimensional (1D) channels within a GaAs heterojunction where a surface acoustic wave (SAW) produces traveling potential wells which convey packets of electrons along the channel [5]. In the SAW pumps, transport of charge resembles the pumping of water by an Archimedean screw. When this principle is combined with Coulomb blockade it results in the pumping of a fixed number of electrons $n$ per cycle. For metrological applications, the delivered current should be in the range of $1 \mathrm{nA}$ and at present only the SAW single-electron pumps satisfy this requirement. However, the accuracy of the SAW pumps must be improved significantly for them to find metrological applications.

A quantum regime of pumping, in which quantum interference plays a key role, was first described by Thouless $[1,2]$. In the Thouless mechanism, a traveling periodic perturbation induces minigaps in the spectrum of an electronic system, and when the Fermi level lies in a minigap an integer number of electrons $n$ are transferred during a cycle, resulting in a quantized current flowing without dissipation. From a fundamental physics standpoint, this mechanism represents a new macroscopic quantum phenomenon reminiscent of the quantum Hall effect and of superconductivity. Possible applications of charge pumping are not limited to metrology. For example, the ability of
PACS numbers: 85.35.Kt, 72.50.+b, 73.23.Hk, 73.63.Kv

the pumps to control the position of single electrons could be used in various quantum information processing schemes [11,12].

Recently it has been pointed out that carbon nanotubes have significant advantages over semiconductor and metallic systems in terms of single-electron pumping [8,9]. The typical Coulomb charging energies achievable in nanotubes can exceed $10 \mathrm{meV}$, significantly larger than in the pumps described in Refs. [3-5,7]. Carbon nanotubes also offer advantages in the quantum regime of pumping due to minigaps an order of magnitude larger being achievable for a given spatial period of the perturbation [8]. Motivated by the potential of nanotubes in this regard, we have undertaken an experimental search for charge pumping in nanotubes by the traveling potential of a SAW. Here we demonstrate that a SAW can indeed pump charge through a carbon nanotube and that the amplitude and polarity of the current can be controlled by a nearby gate electrode. In the final part of the Letter we discuss possible applications of the discovered effects.

We employ the arrangement suggested in Ref. [8] and shown in Fig. 1(a). A nanotube (grown by chemical vapor deposition) lies on a polished $36^{\circ} Y$-cut quartz substrate. Source and drain contacts (separated by $1 \mu \mathrm{m}$ ), a side gate, and a transducer for SAW generation are fabricated by
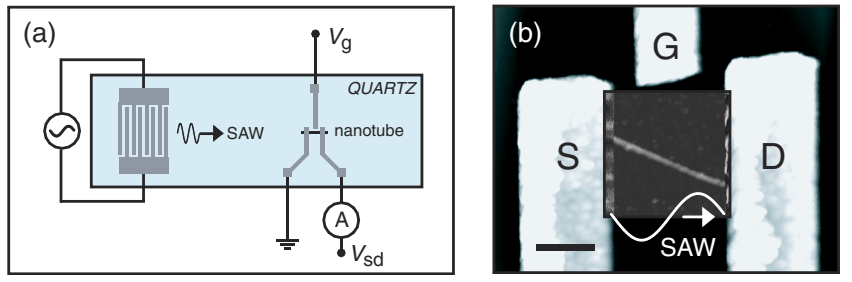

FIG. 1 (color online). (a) Schematic of the device showing the transducer and carbon nanotube. (b) Atomic force microscope image of the contacted nanotube. Scale bar is $500 \mathrm{~nm}$. The gray scale height range is smaller for the central square to highlight the nanotube. The SAW pumps charge from the source $(\mathrm{S})$ to the drain (D), while the carrier type is controlled by the nearby gate (G). 
electron beam lithography. The transducer is $0.7 \mathrm{~mm}$ away from the nanotube and is designed to generate a SAW with a wavelength of $1 \mu \mathrm{m}$ corresponding to a SAW frequency of $3.2 \mathrm{GHz}$. Since quartz is piezoelectric, the SAW is accompanied by an electrostatic potential wave which acts on the electrons in the nanotube. All the data presented here are for a nanotube of diameter $2.5 \pm 0.5 \mathrm{~nm}$ at $T=5 \mathrm{~K}$ $[13,14]$.

Figure 2(a) shows a color scale plot of the current as a function of source-drain bias $\left(V_{\mathrm{sd}}\right)$ and gate voltage $\left(V_{g}\right)$ in the absence of a SAW excitation. A rhomboid-shaped region of low conductance is observed in the center $\left(-5 \mathrm{~V}<V_{g}<1 \mathrm{~V}\right)$, indicating that this nanotube is semiconducting. The slightly higher conductance in the $n$-type regime than in the $p$ type is consistent with the expected difference in Schottky barrier heights for electrons and holes at the Ti contacts [15]. More resolved measurement (not shown) in the $n$ and $p$-type regions show Coulomb blockade oscillations with a charging energy of order 5-10 meV.

Applying rf power $P_{\mathrm{rf}}$ to the transducer induces a dc current $I_{\text {SAW }}$ in the nanotube in the absence of applied bias. The current is generated only within a narrow frequency range, corresponding to the passband of the transducer, see Figs. 3(a) and 3(b). Hence, it occurs only when a SAW is present, and is not a result of rectification of airborne $\mathrm{rf}$ fields. Figure 3(c) shows the variation of $I_{\mathrm{SAW}}$ with $V_{g}$ at $P_{\mathrm{rf}}=20 \mathrm{dBm}(100 \mathrm{~mW})$. At this power level the current is present across the entire gate region in which the low-bias dc conductance is absent, see Fig. 2(b), reversing direction in the center and showing a peak located just outside the insulating region in each direction. At lower power levels

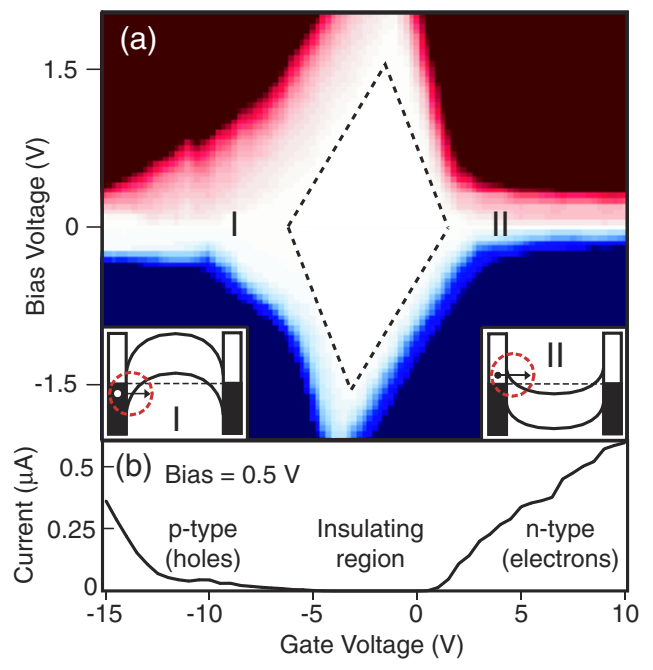

FIG. 2 (color online). (a) Color scale plot of the dc current versus gate and source-drain bias voltage at $T=5 \mathrm{~K}$. Red indicates positive and blue negative currents. The superimposed rhomboid, taken at a threshold current of $0.5 \mathrm{nA}$, gives an indication of the location of the insulating region. Insets: Schematic diagrams showing the difference in Schottky barrier height presented to electrons and holes. (b) Line scan taken at $V_{\text {sd }}=0.5 \mathrm{~V}$. there is a range of $V_{g}$ in which $I_{\mathrm{SAW}}$ is zero, as can be seen in Fig. 4. As the power level decreases this range widens and the peak current in each direction decreases steadily.

For the interpretation of the results in Figs. 2-4 it is convenient to start by observing that the pumped current flows in the insulating state of the nanotube $\left(-5 \mathrm{~V}<V_{g}<\right.$ $1 \mathrm{~V}$ ) when there are no free carriers in the bulk of the nanotube in the absence of the SAW. It implies that carriers must be injected into the nanotube from the source contact by the SAW and, once injected, become trapped in SAW potential minima (or maxima for holes) and are carried along the nanotube by the wave, see Figs. 3(d)-3(f).

Before discussing the implications of a model of charge transport in packets, we note that the observation of charge transport in the insulating state of the nanotube also rules out an alternative transport mechanism in which a SAWinduced rectified voltage would drive a current. A rectification mechanism requires the presence of a region with a nonlinear conductivity. For example, the nonlinearity of the nanotube contacts could be a natural source for a
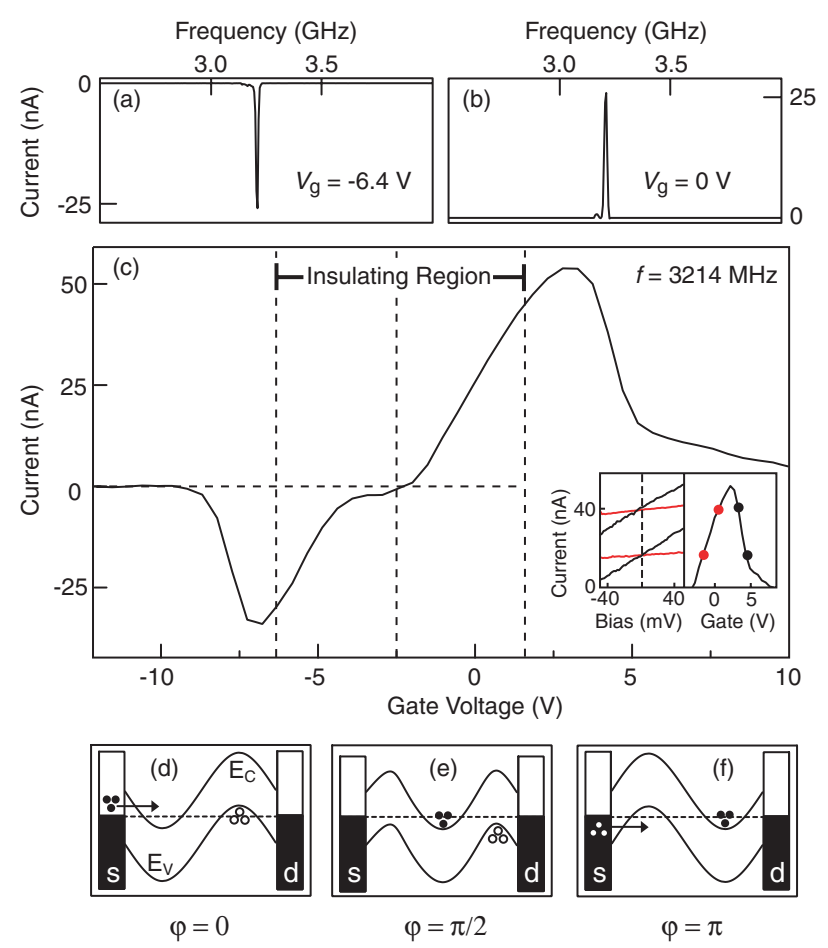

FIG. 3 (color online). (a),(b) Dependence of the SAW-induced current on rf frequency for two values of the gate voltage, showing distinct peaks at the resonant SAW frequency. rf power applied to the transducer is $+20 \mathrm{dBm}$. (c) Dependence of the SAW-induced current on gate voltage. Positive current corresponds to electron flow in the direction of SAW propagation. Inset: Dependence of the current on dc bias for four fixed values of the gate voltage, showing a transition from low to high conductance at the current peak. rf power is $+20 \mathrm{dBm}$. (d)(f) Schematic band diagrams depicting the proposed mechanism of charge transport. The conduction $\left(E_{C}\right)$ and valence $\left(E_{V}\right)$ bands are bent by the SAW allowing injection of electrons or holes depending on the phase $(\phi)$ of the wave [24]. 
rectified dc voltage. The value of such a rectified voltage, however, would not exceed the SAW amplitude which can be estimated from the known geometry of the transducers. We obtain $\Phi_{\mathrm{SAW}} \sim 0.2 \mathrm{~V}$ at $20 \mathrm{dBm}$ of rf power [16]. From Fig. 2 it follows that when the Fermi level is close to the center of the semiconducting gap, a dc voltage above $1 \mathrm{~V}$ is required for a current to flow. This is significantly larger than any possible rectified voltage which could therefore not result in the injection of charge into the nanotube. Moreover, a rectification mechanism could not explain that in our device the current is not sensitive to a source-drain bias [see inset of Fig. 3(c)]. Finally, we note that the sign of the total rectified voltage is not related to the direction of the SAW propagation, while in our experiments the direction of the induced current always followed that of the SAW.

The packet transport model is depicted in Figs. 3(d)3(f). The figures show schematic band diagrams at the moments when the electrons and holes are injected, respectively, for the gate voltage at which the Fermi level is in the center of the semiconducting gap in the absence of the SAW $\left(V_{g} \sim-2.5 \mathrm{~V}\right)$. Here, we neglect the relatively small difference in barrier height for the electrons and holes at the contacts. We also assume that the injection takes place when the SAW electric field is at its maximum at the source contact. The SAW bends the conduction and valence bands of the nanotube so that when the bottom (top) of the conduction (valence) band is below (above) the Fermi level of the source contact, electrons (holes) can tunnel into the nanotube [17]. Thus, the SAW amplitude must exceed a gate voltage dependent threshold value for the injection of charge to be possible. With the Fermi level in the center of the gap, the thresholds for electron and hole injection are the same, and the threshold SAW amplitude is related to the semiconducting gap as $\Phi_{\mathrm{SAW}}($ threshold $)=$ $E_{\text {gap }} / 2 e$. At $20 \mathrm{dBm}$ of rf power applied to the transducer, the SAW amplitude only slightly exceeds the threshold (see Fig. 3) resulting in an estimate of $E_{\text {gap }} \sim 0.4 \mathrm{eV}$. This is in reasonable agreement with the expected value of $0.3 \mathrm{eV}$ for semiconducting nanotubes of diameter $\sim 2.5 \mathrm{~nm}$ [18].

For SAW amplitudes exceeding $\Phi_{\mathrm{SAW}}=E_{\text {gap }} / 2 e$, the current crosses zero at some gate voltage $\left(V_{g} \sim-2.5 \mathrm{~V}\right.$ in Fig. 3). At this value of $V_{g}$ the electron and hole packets contain the same number of particles, resulting in zero net current. If $V_{g}$ is made more positive from this point, the electron packets become bigger than the hole packets so that there is a net positive current. Above some $V_{g}$ the threshold condition for hole injection is not met and the current is carried entirely by electron packets. Likewise, as $V_{g}$ is made more negative, the hole packets become bigger and the net current is negative, and beyond some point it is carried only by holes.

According to the model, pumping should be absent in some interval of $V_{g}$ when the SAW amplitude is below $\Phi_{\text {SAW }}<E_{\text {gap }} / 2 e$. This is indeed the case, as can be seen in Fig. 4 [19]. By adjusting the position of the bands with

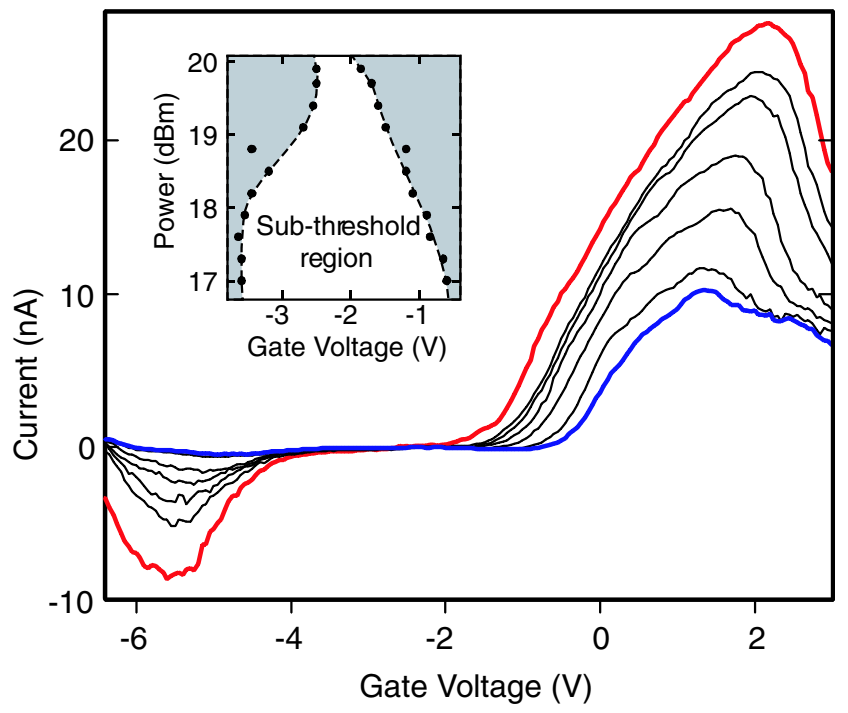

FIG. 4 (color online). SAW-induced current versus gate voltage for fixed values of rf power 17.3 (blue), 17.9, 18.8, 19.1, 19.4, 19.7, and $20 \mathrm{dBm}$ (red). Inset: Points correspond to $\left|I_{\mathrm{SAW}}\right|=$ $0.1 \mathrm{nA}$. This small threshold current was arbitrarily chosen to indicate the region in the $P-V_{g}$ plane in which a SAW-induced current is absent. The dashed lines are a guide to the eye.

respect to the Fermi level (using $V_{g}$ ), one can reach a threshold for the injection of either electrons or holes, but simultaneous transport of both electron and hole packets should not be possible at any $V_{g}$.

Exceeding the threshold is a necessary but not sufficient condition for effective charge injection. A strong enough electric field must also be present at the source contact to make the tunneling barriers transparent for electrons and holes. As the same condition determines the dc bias $V_{\text {sd }}$ required to drive a dc current (Fig. 2) we can compare the SAW electric field to that due to an applied bias voltage. In our device a dc current flows above a bias of $1.5 \mathrm{~V}$ [Fig. 2(a)] giving an average electric field of $\sim 1.5 \mathrm{~V} / \mu \mathrm{m}$ along the nanotube. The maximum electric field due to the SAW at $P_{\text {rf }}=+20 \mathrm{dBm}$ is indeed close to this value at $E_{\max }=2 \pi \Phi_{\mathrm{SAW}} / \lambda \sim 1.3 \mathrm{~V} / \mu \mathrm{m}$. We note that the actual electric field (caused either by dc bias or by the SAW) can be significantly enhanced in a region very close to a thin contact strip due to geometrical considerations.

The maximum number, $n_{\max }$, of electrons (holes) that can be contained by a SAW potential well is given approximately by $n_{\max }=C \Phi_{\mathrm{SAW}} / e$, where $C$ is the self capacitance of a section of the nanotube containing the charge packet. Given a ratio $e / C \sim 5 \mathrm{mV}$, expected for a carbon nanotube of $1 \mu \mathrm{m}$ [20], we arrive at $n_{\max } \sim 40$, corresponding to $I_{\mathrm{SAW}}=n_{\max } e f \sim 20 \mathrm{nA}$ for $f=3.2 \mathrm{GHz}$. Thus, we can justify currents up to this order as occurring by the transport mechanism described.

We have until now limited our discussion of the SAWinduced current to the range of $V_{g}$ in which the dc conductance is zero. Outside this range the current shows distinct peaks, after which it drops dramatically, Fig. 3(c). We 
suggest that outside the insulating region the free carriers screen the SAW field, the carriers are delocalized, and the description of transport in charge packets does not apply. In this "drag" regime the dc current in the nanotube is proportional to the momentum transfer from the wave to the nanotube's electronic system. If we assume, by analogy with the situation in a two-dimensional electron gas $[21,22]$, that the momentum transfer decreases when the nanotube's conductivity exceeds some value, then it explains the decrease of the current with $V_{g}$ in Fig. 3. In this picture, the peaks in current in Fig. 3(c) manifest a crossover from the transport in packets to the drag-type transport regime. This assertion about the change of the transport mechanism can be tested since the charge packet transport should be little affected by a dc bias, whereas outside the insulating region when free carriers exist in the nanotube, the effect of a bias is expected to be much stronger. Such behavior is indeed observed as shown in the inset of Fig. 3(c).

We conclude with a discussion on the feasibility of pumping single electrons in carbon nanotubes. Whereas the Thouless mechanism is more likely to be observed in metallic nanotubes, the classical mechanism of SAW single-electron pumping should be observable for the semiconducting nanotubes studied here. In that mechanism the number of electrons in a packet is fixed within an interval of values of the relevant parameters, such as gate voltage or SAW amplitude, due to Coulomb interactions. This work shows that transport of charge in the form of packets can be realized in nanotubes. For single-electron pumping each packet (or moving quantum dot) should be populated with the same number of electrons and the conditions at the source contact where the SAW potential well is filled with electrons (holes) become crucial.

In particular, the time for electrons (holes) to enter the moving SAW dot should be long compared to the $R C_{\mathrm{cd}}$ time of the junction, where $R$ is a contact resistance and $C_{\mathrm{cd}}$ is the capacitance between the contact and the dot. As an order of magnitude estimate of the tunneling rate we take values $R \sim 10 \mathrm{M} \Omega$ and $C_{\mathrm{cd}} \sim 10 \mathrm{aF}$, as deduced from measurements of the device in the Coulomb blockade regime. This yields an inverse $R C$ time of order $10 \mathrm{GHz}$ which only slightly exceeds the SAW frequency of $3.2 \mathrm{GHz}$ and implies that the number of electrons tunneling into a SAW dot fluctuates from cycle to cycle. The conditions at the source contact can be improved by using a better (less resistive) source contact, such as Pd [23]. An alternative solution would be to induce electrons or holes in a section of a nanotube adjacent to the source electrode with the help of an additional side gate. Then, as with the GaAs SAW pumps [5], the filling of a SAW potential well with carriers will not involve a slow tunneling process and the quantized transport regime should be observable.

The authors acknowledge stimulating discussions with L. S. Levitov, D. S. Novikov, B. D. Simons, B. Spivak, and D. J. Thouless. This work was supported by the EPSRC UK
(Grant No. GR/R67521) and the EC program SAWPHOTON. V. T. and P.L. acknowledge support from the Newton Trust and the National Physical Laboratory, respectively.

[1] D. J. Thouless, Phys. Rev. B 27, 6083 (1983).

[2] Q. Niu and D. J. Thouless, J. Phys. A 17, 2453 (1984).

[3] L. J. Geerligs et al., Phys. Rev. Lett. 64, 2691 (1990).

[4] L.P. Kouwenhoven et al., Phys. Rev. Lett. 67, 1626 (1991).

[5] V. I. Talyanskii et al., Phys. Rev. B 56, 15180 (1997).

[6] M. Switkes et al., Science 283, 1905 (1999).

[7] M.W. Keller, A.L. Eichenberger, J.M. Martinis, and N. M. Zimmerman, Science 285, 1706 (1999).

[8] V. I. Talyanskii, D.S. Novikov, B. D. Simons, and L.S. Levitov, Phys. Rev. Lett. 87, 276802 (2001).

[9] P. Sharma and C. Chamon, Phys. Rev. Lett. 87, 096401 (2001).

[10] J. Ebbecke, C. J. Strobl, and A. Wixforth, Phys. Rev. B 70, 233401 (2004).

[11] D. P. DiVincenzo and C. Bennett, Nature (London) 404, 247 (2000).

[12] C. H. W. Barnes, J. M. Shilton, and A. M. Robinson, Phys. Rev. B 62, 8410 (2000).

[13] Given the relatively large diameter, it cannot be excluded that the nanotube actually consists of a small bundle, or a multiwalled nanotube. However, the characteristic rhomboid shown in Fig. 2 suggests that we are dealing with a single nanotube.

[14] Similar data to those reported here have been measured on two other devices.

[15] V. Derycke, R. Martel, J. Appenzeller, and Ph. Avouris, Appl. Phys. Lett. 80, 2773 (2002).

[16] R. L. Miller, C.E. Nothnic, and D.S. Bailey, Acoustic Charge Transport: Device Technology and Applications (Artech House, Boston, 1992).

[17] We estimate the band bending due to a mechanical perturbation of the nanotube by the SAW to be much smaller than that due to the SAW electrostatic field.

[18] J. W. G. Wildöer, L.C. Venema, A. G. Rinzler, R. E. Smalley, and C. Dekker, Nature (London) 391, 59 (1998).

[19] There is a slight difference between $I_{\mathrm{SAW}}\left(V_{g}\right)$ graphs for $P=+20 \mathrm{dBm}$ in Figs. 3 and 4 . We attribute this to a minor change in transducer resonant frequency that resulted in a small decrease of SAW amplitude for the graph in Fig. 4. The presented interpretation of the data is unaffected.

[20] P. L. McEuen, M. Bockrath, D. H. Cobden, Y.-G. Yoon, and S. G. Louie, Phys. Rev. Lett. 83, 5098 (1999).

[21] G. Weinreich, Phys. Rev. 104, 321 (1956).

[22] A. Wixforth, J. P. Kotthaus, and G. Weimann, Phys. Rev. Lett. 56, 2104 (1986).

[23] A. Javey, J. Guo, Q. Wang, M. Lundstrom, and H. Dai, Nature (London) 424, 654 (2003).

[24] The source and drain potentials are effectively grounded at SAW frequencies. This distorts the bands close to the contacts when the SAW potential is nonzero, affecting panel $E$, but not panels $D$ and $F$. 